\title{
Construction of an Effective Preconditioner for the Even-odd Splitting of Cubic Spline Wavelets
}

\author{
BORIS M. SHUMILOV \\ Department of Applied Mathematics \\ Tomsk State University of Architecture and Building \\ 2 Solyanaya, Tomsk, 634003 \\ RUSSIA
}

\begin{abstract}
In this study, the method for decomposing splines of degree $m$ and smoothness $C^{m-1}$ into a series of wavelets with zero moments is investigated. The system of linear algebraic equations connecting the coefficients of the spline expansion on the initial scale with the spline coefficients and wavelet coefficients on the embedded scale is obtained. The originality consists in the application of some preconditioner that reduces the system to a simpler band system of equations. Examples of applying the method to the cases of first-degree spline wavelets with two first zero moments and cubic spline wavelets with six first zero moments are presented. For the cubic case after splitting the system into even and odd rows, the resulting matrix acquires a seven-diagonals form with strict diagonal dominance, which makes it possible to apply an effective sweep method to its solution.
\end{abstract}

Key-Words: - $B$-splines, wavelets, implicit decomposition relations, sweep method, data processing

Received: July 28, 2021. Revised: December 2, 2021. Accepted: December 19, 2021. Published: December 28, 2021.

\section{Introduction}

A wavelet is a short or rapidly decaying wave function (burst), for which a set of contractions and displacements generates a space of measurable functions on the entire number axis [1-4]. Because of compressions, the wavelets reveal varying scales of detail differences between the characteristics of the measured signal, and due to shifts, they can analyze the properties of a signal at different points throughout the studied interval. Unlike Fourier transform, which provides only amplitude-frequency information but loses time information, discrete wavelet transform analyses the signal in a time-frequency domain using windows of variable sizes, relative to the frequency band; therefore minimizing the spectral leakage [5]. In solving problems of numerical analysis, since wavelets transform a system of basic functions with distributed parameters into a direct sum of systems with lumped parameters, such a basis is more efficient in terms of stability and convergence [6]. For example, effective denoising methods are required for processing measured signals, because conventional denoising methods may not be practical due to the noisy nature of the measuring as well as its spectral overlap with different noise sources. The papers $[7,8]$ propose an adaptive technique based on wavelets and artificial neural networks to filtrate signals in a noisy environment. With multimedia becoming widely popular, the conflict between mass data and finite memory devices has been continuously intensified; so, it requires more convenient, efficient, and high-quality transmission and storage technology, and the fast wavelet analysis is what people want to use for highly efficient compression technology [9]. At the same time, the multiscale color wavelet texture feature detection method has been widely used to improve the segmentation accuracy of medical images [10], such as retinal fundus images [11], or electrocardiogram (ECG) signals [12].

The basis for constructing wavelets is the presence of a set of approximating spaces $\ldots V_{L-1} \subset V_{L} \subset$ $V_{L+1} \ldots$ such that each basis function in $V_{L}$ can be expressed as a linear combination of basis functions in $V_{L+1}$. In particular, splines - smooth functions glued from pieces of polynomials of degree $m$ on an embedded sequence of grids, have this property. The essence of the wavelet transform can be formulated as follows: it allows one to decompose a given function $V_{L+1}$ into a rough approximate representation $V_{L}$ and local refinements $W_{L}=V_{L+1}-V_{L}$. The procedure for dividing into a rough version and clarifying details can be applied recursively to this part of $V_{L}$. Hence, the original function can be represented as a hierarchy of rough versions of $V_{L}, V_{L-1}, \ldots$ and refinements $W_{L}, W_{L-1}, \ldots$. Such a recursive process is called direct wavelet transformation (decomposition or analysis) [4, p. 46]. Conversely, the function $V_{L+1}$ can be reconstructed from the most compact representation (reconstruction). Moreover, the values of the coefficients of the wavelet decomposition can be used to judge the significance of the corresponding 
details of the refinement. Insignificant details can be removed to compress the information. The main thing here is to find a suitable basis for the space $W_{L}$ and construct for it fast one-to-one formulas for the direct and inverse wavelet transforms.

\section{Related work}

Ingrid Daubechies was the first to construct orthonormal wavelets of non-polynomial type with compact support [1]. Cohen et al constructed biorthogonal wavelets $[3,13,14]$. The disadvantages of these wavelets are that they have no analytical representation and are graphically similar to fractal curves. Both properties are extremely important when used for the approximate solution of problems of numerical analysis. As to spline wavelets, there are various possibilities for constructing basic functions in space $W_{L}$. For example, as basic functions in space $W_{L}$, we can use orthogonal wavelets $[3$, p. 103] elements of the space $V_{L+1}$, orthogonal to all elements of the space $V_{L}$, and among themselves, or semi-orthogonal wavelets [3, p. 107]-elements of the space $V_{L+1}$, orthogonal to all elements of the space $V_{L}$, but not orthogonal to each other. In both cases, the wavelet decomposition provides the best root-meansquare approximation of splines on a dense grid using splines on a sparse grid [15]. The difference between orthogonal and semi-orthogonal wavelets consists in the presence or absence of explicit finite decomposition formulas and, conversely, in the absence or presence of explicit analytical formulas for calculating wavelets. The only wavelet that fully satisfies all of these properties is the Haar wavelet, the disadvantage of which is that being an element of the spline space of degree zero it has discontinuities at the nodes. There are other wavelets for which there are explicit finite decomposition formulas, such as "lazy" wavelets [3] and their generalizations in the form of biorthogonal wavelets $[13,14]$. The disadvantage of these wavelets is that the expansion coefficients are equal to the values of the function in some nodes of a dense grid, or are calculated using local averaging formulas, that is, the information for calculating each coefficient is not fully used. In turn, the interpolating spline wavelets [16] are calculated by solving interpolation problems on a sequence of nested grids, providing the best root-mean-square approximation of the derivative of the order $(m+1) / 2$, but the function values are not filtered at all, representing a kind of data thinning. In contrast, the coefficients of semiorthogonal [3, p. 115] wavelets are connected by systems of linear algebraic equations, but the good conditionality of the systems is not guaranteed.

The unique properties of wavelets allow designing a basis in which the data representation can be expressed with a small number of non-zero coeffi- cients. This property makes wavelets attractive for data compression, including video and audio information. Wavelet transform can be viewed as one of the methods of primary signal processing to improve the efficiency of its compression. In this case, direct compression is performed by classical methods only for significant coefficients of the wavelet decomposition of the signal, and its reconstruction according to these coefficients is performed at the stage of restoration (decompression) [17]. For the indicated problem, it is important to complement smoothly the discretely given functions. Not all types of wavelets meet these goals, but only some of them, for example, wavelets based on $B$-splines and Hermitian splines [18]. In particular, Chui et al constructed semi-orthogonal spline wavelets [4]. The disadvantage of these wavelets is that they have rather large supports.

Meanwhile, in the work of the author [19], nonorthogonal wavelets of the third degree with the first four zero moments, that is, orthogonal to all polynomials of the third degree, were considered; the existence of finite implicit decomposition relations was proved, and an effective algorithm for wavelet analysis based on them was justified. The importance of the new algorithm in wavelets theory is its stability and the simplicity of realization because a matrix possessing strict diagonal prevalence is solved at each step of resolution.

This article attempts to find out if there is an even-odd splitting algorithm for non-orthogonal cubic wavelets with the six first zero moments, and a strict diagonal dominance of the splitting systems is proved. Section 3 discusses the properties of the splines of degree $m$ of smoothness $C^{m-1}$ on a uniform infinite grid of nodes and the non-orthogonal spline-wavelets with $n+1$ vanishing moments. Section 4 considers the use of the matrix notation to wavelet transformation of hierarchical spline bases and suggests the main idea of preconditioning the wavelet transform system of equations. As a result, in section 5 the case of spline-wavelets of 3-rd degree with 6 zero moments is fully resolved and in section 6 the wavelet interpolation algorithm on the finite segment is implemented. Here a strict diagonal dominance of the resulting system is also proved, which assures the stability of the calculations with the sweep method. In the seventh section, the algorithm of wavelet analysis is presented as a whole, and section 8 contains examples of the application.

\section{Construction of spline wavelets with vanishing moments}

Let the space $V_{L}$ be the space of splines of degree $m$ of smoothness $C^{m-1}$ on a uniform grid of nodes 
$\Delta^{L}: x_{i+1}=x_{i}+1 / 2^{L}$, infinitely extended in both directions for all $i$. It is well known that the basis in this space is generated by functions $\varphi_{m}(v-i) \forall i$, where $v=2^{L} x$, generated by contractions and shifts of a function of the form [20, p. 89]:

$$
\varphi_{m}(t)=\frac{1}{m !} \sum_{j=0}^{m+1}(-1)^{j}\left(\begin{array}{c}
m+1 \\
j
\end{array}\right)(t-j)_{+}^{m},
$$

where $t_{+}^{m}=(\max \{t, 0\})^{m}$.

They have the following supports,

$$
\operatorname{supp} \varphi_{m}=[0, m+1],
$$

and they satisfy the calibration relation [4, p. 91]:

$$
\varphi_{m}(t)=2^{-m} \sum_{k=0}^{m+1}\left(\begin{array}{c}
m+1 \\
k
\end{array}\right) \varphi_{m}(2 t-k) .
$$

As a result, any spline on the mesh $\Delta^{L}$ can be represented as

$$
s^{L}(x)=\sum_{-\infty}^{\infty} c_{i}^{L} \varphi_{m}\left(2^{L} x-i\right),
$$

where the coefficients $c_{i}^{L} \forall i$ are the solution, for example, of the cardinal interpolation problem:

$$
s^{L}\left(x_{i}\right)=f\left(x_{i}\right),-\infty<i<\infty .
$$

If the grid $\Delta^{L-1}$ is obtained from the grid $\Delta^{L}$ by removing every second node, then the corresponding space $V_{L-1}$ with basic functions $\varphi_{m}(v / 2-i) \forall i$, whose supports are twice as wide in width, is embedded in $V_{L}$. The difference between the spaces $V_{L}$ and $V_{L-1}$ constitutes the wavelet space $W_{L-1}=$ $V_{L}-V_{L-1}[4]$.

The supports of the orthogonal wavelets are rigidly coupled with the supports of the basic splines. In contrast, in what follows we will use non-orthogonal wavelets with $n+1$ vanishing moments, that is, orthogonal to all polynomials of degree $n$ [21, p. 157],

$$
w_{m, n}(t)=2^{-m} \sum_{k=0}^{n+1}(-1)^{k}\left(\begin{array}{c}
n+1 \\
k
\end{array}\right) \varphi_{m}(2 t-k) \text {. }
$$

These functions have the following supports

$$
\operatorname{supp} w_{m, n}=\left[0, \frac{m+n}{2}+1\right]
$$

and, accordingly, $n+1$ zero moments

$$
\int_{-\infty}^{\infty} x^{k} w_{m, n}(x) d x=0, k=0,1, \ldots, n .
$$

In addition, they can be moved, within certain limits, relative to the supports of the basic splines. For the

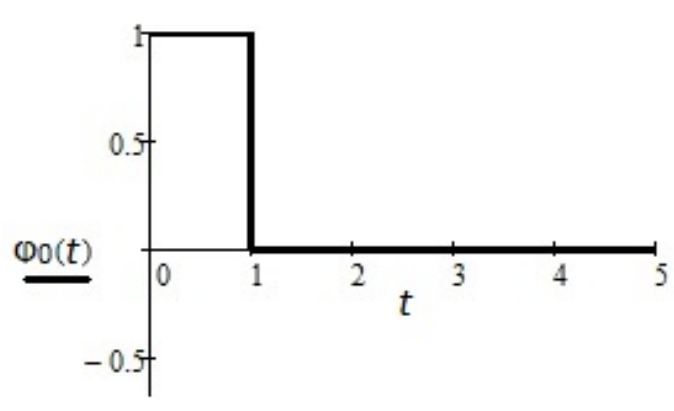

Figure 1: The graph of the scaling function $\varphi_{0}(t)$

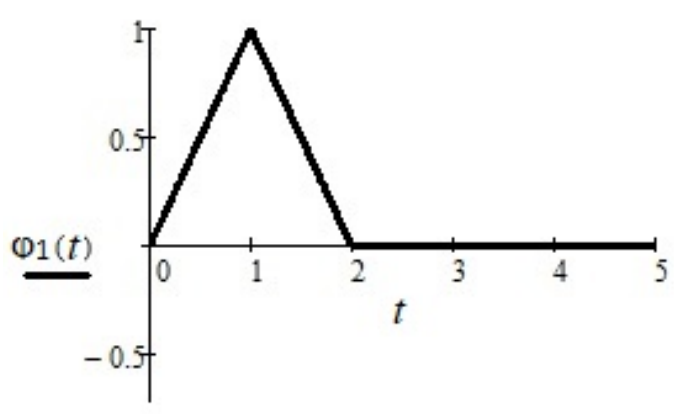

Figure 2: The graph of the scaling function $\varphi_{1}(t)$

cases $m=0,1,3$, the basis spline functions take on forms (Fig. 1-3)

The graphs of wavelets of the $0,1,3$ rd degrees, orthogonal to all polynomials of the $0,1,5$ th degrees, are shown in figures 4-6.

\section{Construction of the defining system of wavelet transform equations}

We write the basic spline functions in the form of a one-row matrix of infinite length,

$$
\varphi^{L}(x)=\left[\ldots, \varphi_{m}\left(2^{L} x-i\right), \varphi_{m}\left(2^{L} x-i-1\right), \ldots\right] .
$$

Introducing the notation

$$
\mathbf{c}^{L}=\left[\ldots, c_{i}^{L}, c_{i+1}^{L}, \ldots\right]^{T}
$$

for a vector consisting of the spline coefficients, we 


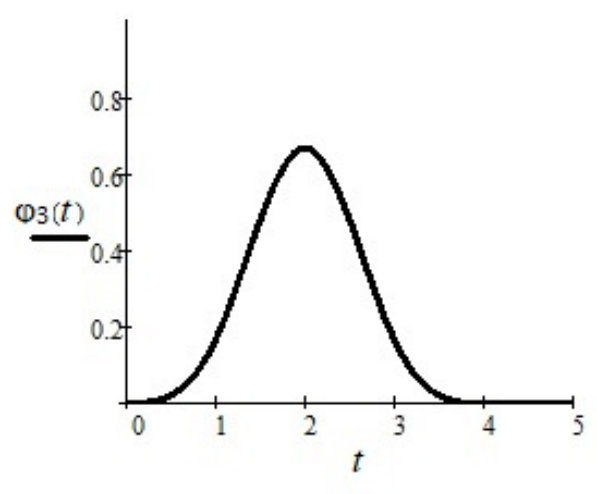

Figure 3: The graph of the scaling function $\varphi_{3}(t)$

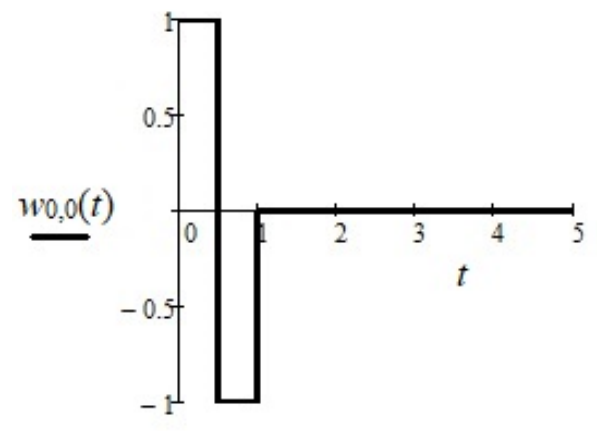

Figure 4: The graph of the function $w_{0,0}(t)$

write the formula (2) in a vector form

$$
s^{L}(x)=\varphi^{L}(x) \mathbf{c}^{L} .
$$

In the same way, we can write basic wavelet functions at the decomposition level $L$ in the form of an infinite row matrix as

$$
\begin{gathered}
\psi^{L-1}(x)= \\
=\left[\ldots, w_{m, n}\left(2^{L} x-i\right), w_{m, n}\left(2^{L} x-i-1\right), \ldots\right] .
\end{gathered}
$$

We denote the corresponding wavelet approximation coefficients by $d_{i}^{L-1},-\infty<i<\infty$, and introduce the column vector

$$
\mathbf{d}^{L-1}=\left[\ldots, d_{i}^{L-1}, d_{i+1}^{L-1}, \ldots\right]^{T} .
$$

Since the spaces $V_{L-1}$ and $W_{L-1}$ are by definition subspaces of $V_{L}$, the functions $\varphi^{L-1}(x)$ and $\psi^{L-1}(x)$ can be represented as linear combinations of the functions $\varphi^{L}(x)$ :

$$
\varphi^{L-1}(x)=\varphi^{L}(x) P^{L} \text { and } \psi^{L-1}(x)=\varphi^{L}(x) Q^{L},
$$

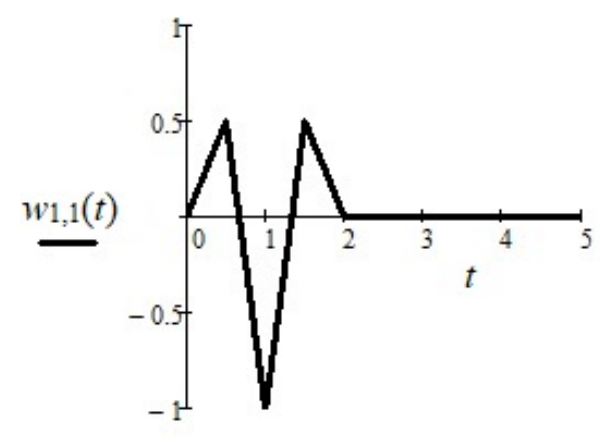

Figure 5: The graph of the function $w_{1,1}(t)$

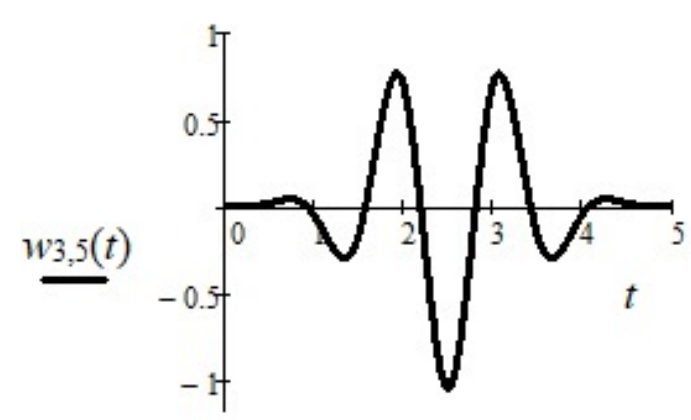

Figure 6: The graph of the function $w_{3,5}(t)$

where the columns of the matrix $P^{L}$ are composed of the relation coefficients (1) since each wide basic function can be constructed from $m+2$ narrow basic functions, while the elements of the columns of the matrix $Q^{L}$ are composed of the relation coefficients (3).

Therefore, there is a chain of equalities:

$$
\begin{aligned}
\varphi^{L}(x) \mathbf{c}^{L} & =\varphi^{L-1}(x) \mathbf{c}^{L-1}+\psi^{L-1}(x) \mathbf{d}^{L-1}= \\
& =\varphi^{L}(x) P^{L} \mathbf{c}^{L-1}+\varphi^{L}(x) Q^{L} \mathbf{d}^{L-1} .
\end{aligned}
$$

Let the coefficients $\mathbf{c}^{L-1}$ and $\mathbf{d}^{L-1}$ be known. Then the coefficients $\mathbf{c}^{L}$ can be easily obtained from $\mathbf{c}^{L-1}$ and $\mathbf{d}^{L-1}$ as follows

$$
\mathbf{c}^{L}=P^{L} \mathbf{c}^{L-1}+Q^{L} \mathbf{d}^{L-1} .
$$

Formula (4) is nothing more than a recovery algorithm [4, p. 248], for the implementation of which, due to tape matrices $P^{L}$ and $Q^{L}$ the moving average scheme is successfully used. For example, for 
$m=n=0$ (the case of Haar wavelets) the formula (4) takes the form

$$
\begin{aligned}
c_{2 i}^{L} & =c_{i}^{L-1}+d_{i}^{L-1}, \\
c_{2 i+1}^{L} & =c_{i}^{L-1}-d_{i}^{L-1},-\infty<i<\infty,
\end{aligned}
$$

while for $m=n=1$ (the case of splines and wavelets of the first degree) the formula (4) takes the form

$$
\begin{aligned}
c_{2 i}^{L} & =\frac{1}{2}\left(c_{i}^{L-1}+c_{i+1}^{L-1}+d_{i}^{L-1}+d_{i+1}^{L-1}\right), \\
c_{2 i+1}^{L} & =c_{i+1}^{L-1}-d_{i+1}^{L-1},-\infty<i<\infty .
\end{aligned}
$$

Unfortunately, for the reverse process of calculating from the coefficients $\mathbf{c}^{L}$, the coarser version $\mathbf{c}^{L-1}=A^{L} \mathbf{c}^{L}$ and the refining coefficients $\mathbf{d}^{L-1}=$ $B^{L} \mathbf{c}^{L}$, following the decomposition algorithm [4, p. 247], for all cases, except for the case of Haar wavelets, when due to their orthorgonality

$$
\begin{aligned}
c_{i}^{L-1} & =\frac{1}{2}\left(c_{2 i}^{L}+c_{2 i+1}^{L}\right), \\
d_{i}^{L-1} & =\frac{1}{2}\left(c_{2 i}^{L}-c_{2 i+1}^{L}\right),-\infty<i<\infty,
\end{aligned}
$$

we obtain that the rows of matrices $A^{L}$ and $B^{L}$ are infinite numerical sequences, and their truncation leads to errors.

Using the notation for block matrices, we rewrite equality (4) in the form

$$
\mathbf{c}^{L}=\left[P^{L} \mid Q^{L}\right]\left[\frac{\mathbf{c}^{L-1}}{\mathbf{d}^{L-1}}\right]
$$

and define some infinite matrices $R^{L}$ and $G^{L}$ by the conditions:

a) $G^{L}=\left[P^{L} \mid Q^{L}\right] R^{L}$;

b) matrix $R^{L}$ - tape matrix, with the minimum possible number of elements;

c) $G^{L}$ is a tape matrix with the minimum possible number of nonzero diagonals.

Then, assuming that the matrix $G^{L}$ is nondegenerate, we multiply the left and right sides of equality (5) by the matrix $R^{L} G^{L^{-1}}$. As a result, we get

$$
\begin{gathered}
R^{L} G^{L^{-1}} \mathbf{c}^{L}= \\
=R^{L}\left(\left[P^{L} \mid Q^{L}\right] R^{L}\right)^{-1}\left[P^{L} \mid Q^{L}\right]\left[\frac{\mathbf{c}^{L-1}}{\mathbf{d}^{L-1}}\right]= \\
=R^{L} R^{L^{-1}}\left[P^{L} \mid Q^{L}\right]^{-1}\left[P^{L} \mid Q^{L}\right]\left[\frac{\mathbf{c}^{L-1}}{\mathbf{d}^{L-1}}\right] .
\end{gathered}
$$

Thus, instead of directly solving a system of the form (5), we can solve the system

$$
G^{L} \mathbf{h}^{L}=\mathbf{c}^{L}
$$

with respect to some values of $\mathbf{h}^{L}$ and then just calculate the values of $\mathbf{c}^{L-1}$ and $\mathbf{d}^{L-1}$ using the linear transformation

$$
\left[\frac{\mathbf{c}^{L-1}}{\mathbf{d}^{L-1}}\right]=R^{L} \mathbf{h}^{L} .
$$

In order to illustrate the application of this technology to the specific example of splines and wavelets of the first degree, let us follow to [22].

Let the system (7) compounds from the equations

$$
\begin{aligned}
c_{i}^{L-1}= & \frac{1}{2}\left(2 h_{2 i-1}^{L}+h_{2 i}^{L}+2 h_{2 i+1}^{L}\right), \\
d_{i}^{L-1}= & \frac{1}{2}\left(2 h_{2 i-1}^{L}-h_{2 i}^{L}+2 h_{2 i+1}^{L}\right), \\
& -\infty<i<\infty .
\end{aligned}
$$

Then, multiplying the matrises $\left[P^{L} \mid Q^{L}\right]$ and $R^{L}$ we obtain that the matrix $G^{L}$ has three nonempty diagonals with elements

$$
\begin{aligned}
& \{\cdots 0101010 \cdots\}, \\
& \{\cdots 000000000 \cdots\} \text {, } \\
& \{\cdots 1212121 \cdots\} \text {, } \\
& \{\cdots 000000000 \cdots\} \text {, } \\
& \{\cdots 0101010 \cdots\} \text {, }
\end{aligned}
$$

from which and (6) it immediately follows, that

$$
h_{2 i}^{L}=c_{2 i}^{L}, \forall i,
$$

while odd elements of $\mathbf{h}^{L}$ been connected by the infinite three-diagonals system of equations

$$
h_{2 i-1}^{L}+2 h_{2 i+1}^{L}+h_{2 i+3}^{L}=c_{2 i+1}^{L}, \forall i .
$$

As a result, the wavelet interpolation algorithm on a finite segment $\left[1,2^{L}\right]$ consists of resetting the value of the observation result at the first point to zero by subtracting its value from the entire time series. The system (8) is closed by the bounding equations

$$
\begin{aligned}
h_{1}^{L}+h_{3}^{L} & =c_{1}^{L}, \\
h_{i-3}^{L}+2 h_{i}^{L} & =c_{i}^{L}, i=2^{L}-1 .
\end{aligned}
$$

And after wavelet analysis of the differences obtained and reconstructing (of course, with some approximation) the spline coefficients for the densest mesh, the value of the first observation are added to values of the approximating spline of the first degree.

\section{Spline-wavelets of 3-rd degree with 6 zero moments}

For the case $m=3, n=5$, the infinite matrix $\left[P^{L} \mid Q^{L}\right]$ takes the following form:

$$
\left[P^{L} \mid Q^{L}\right]=\frac{1}{8} \text {. }
$$




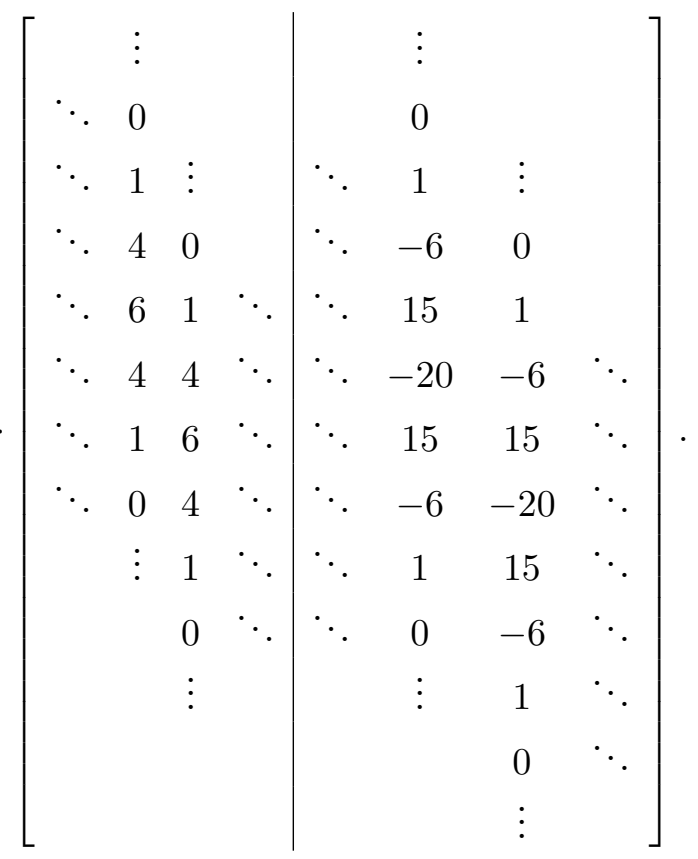

For the matrix $\left[P^{L} \mid Q^{L}\right]$, the seven-diagonals matrix

$$
\left[P^{L} \mid Q^{L}\right]^{\prime}=\frac{1}{8} \text {. }
$$

$$
\left[\begin{array}{ccccccccc}
\ddots & \ddots & \vdots & \vdots & & & & & \\
\ddots & -6 & 0 & 0 & \vdots & & & & \\
\ddots & 15 & 1 & 1 & 0 & \vdots & & & \\
\ddots & -20 & 4 & -6 & 0 & 0 & \ddots & & \\
\ddots & 15 & 6 & 15 & 1 & 1 & 0 & \cdots & \\
\ddots & -6 & 4 & -20 & 4 & -6 & 0 & 0 & \cdots \\
\ddots & 1 & 1 & 15 & 6 & 15 & 1 & 1 & \ddots \\
\cdots & 0 & 0 & -6 & 4 & -20 & 4 & -6 & \ddots \\
\cdots & 0 & 0 & 1 & 1 & 15 & 6 & 15 & \ddots \\
& \ddots & 0 & 0 & 0 & -6 & 4 & -20 & \ddots \\
& & \vdots & 0 & 0 & 1 & 1 & 15 & \ddots \\
& & & \vdots & 0 & 0 & 0 & -6 & \ddots \\
& & & & \vdots & \vdots & \ddots & \ddots & \ddots
\end{array}\right]
$$

is obtained by permuting the columns of the matrix $\left[P^{L} \mid Q^{L}\right]$ so that the columns of the matrices $P^{L}$ and $Q^{L}$ alternate. In practice, such a permutation is accompanied by a change in the order of the unknowns in the system (5) and is often done in order to give the system a tape-like form to facilitate the numerical solution of the system [3]. However, as can be seen from the above example, the resulting system of equations does not have a diagonal dominance, which can complicate the wavelet analysis of large data.
The matrix corresponding to the indicated permutation of columns is denoted by $T$. Then the representation is true [23]

$$
\left[P^{L} \mid Q^{L}\right]^{\prime}=\left[P^{L} \mid Q^{L}\right] T .
$$

From the representation (9) we find

$$
\left[P^{L} \mid Q^{L}\right]^{-1} \cdot G^{L}=T \cdot\left[P^{L} \mid Q^{L}\right]^{\prime-1} \cdot G^{L} .
$$

Thus, the problem was reduced to finding a solution to the system of matrix equalities

$$
\left[P^{L} \mid Q^{L}\right]_{j}^{\prime} R_{j}^{L^{\prime}}=G_{j}^{L},-\infty<j<\infty .
$$

Here, the subscripts in the notation of the matrices indicate which elements of the columns of the matrix $R^{L^{\prime}}$ are calculated by the corresponding system (11). By placing as many zeros as possible in the upper and lower parts of each column of the matrix $R^{L^{\prime}}$, we ensure the compactness of the computational scheme; by zeroing the elements spaced by an odd number of steps from the main diagonal of the matrix $G^{L}$, we provide the possibility of using an efficient sweep method for solving it; and additionally zeroing the elements outside the main diagonal of the matrix $G^{L}$, we provide the possibility of the subsequent splitting of the system into even and odd rows. Earlier in [24], we proved that the matrix for a 5-diagonals splitting does not have a diagonal dominance. Therefore, here we consider the case of a 7-diagonasl splitting. Specifically, according to the assumed stepped structure of the matrices $R^{L^{\prime}}$ and $G^{L}$, system (10) splits into blocks with matrices of the following form:

$$
\left[P^{L} \mid Q^{L}\right]_{j, j+1, \ldots, j+8}^{\prime}=\frac{1}{8} .
$$

$$
\left[\begin{array}{ccccccccc}
1 & 1 & 0 & 0 & & & & & \\
4 & -6 & 0 & 0 & & & & & \\
6 & 15 & 1 & 1 & & & & & \\
4 & -20 & 4 & -6 & & & & & \\
1 & 15 & 6 & 15 & 1 & 1 & & & \\
0 & -6 & 4 & -20 & 4 & -6 & & & \\
& 1 & 1 & 15 & 6 & 15 & 1 & 1 & \\
& 0 & 0 & -6 & 4 & -20 & 4 & -6 & \\
& & & 1 & 1 & 15 & 6 & 15 & 1 \\
& & & 0 & 0 & -6 & 4 & -20 & 4 \\
& & & & & 1 & 1 & 15 & 6 \\
& & & & & 0 & 0 & -6 & 4 \\
& & & & & & & 1 & 1
\end{array}\right]
$$

provided that the equations corresponding to the zero rows of the matrix $G^{L}$ are removed from the system. 
This system is solvable and underdetermined. Therefore, we can choose any non-trivial solutions that interest us, for example:

1)

$$
\begin{gathered}
r_{0}=r_{8}=9 ; r_{1}=r_{7}=6 ; \\
r_{2}=r_{6}=0 ; r_{3}=r_{5}=-14 ; \\
r_{4}=-82 ; g_{0}=g_{12}=15 ; \\
g_{1}=g_{3}=g_{5}=g_{7}=g_{9}=g_{11}=0 ; \\
g_{2}=g_{10}=130 ; \\
g_{4}=g_{8}=-207 ; g_{6}=-900 ;
\end{gathered}
$$

2) $r_{0}=r_{1}=r_{5}=r_{6}=r_{7}=r_{8}=0$;

$$
\begin{gathered}
r_{2}=r_{3}=3 ; r_{4}=2 ; \\
g_{0}=g_{1}=g_{3}=g_{7}=g_{9}= \\
=g_{10}=g_{11}=g_{12}=0 ; \\
g_{2}=g_{8}=5 ; \\
g_{4}=g_{6}=51 ; g_{5}=-16 ;
\end{gathered}
$$

3) $r_{0}=r_{1}=r_{2}=r_{3}=r_{7}=r_{8}=0$;

$$
\begin{gathered}
r_{4}=r_{6}=3 ; r_{5}=2 ; \\
g_{0}=g_{1}=g_{2}=g_{3}=g_{5}= \\
=g_{9}=g_{11}=g_{12}=0 ; \\
g_{4}=g_{10}=5 ; \\
g_{6}=g_{8}=51 ; g_{7}=-16 .
\end{gathered}
$$

As a result, the matrix $G^{L}$ acquires a tape structure with eleven nonzero diagonals of the form

$$
\left[\begin{array}{ccccccc}
\ddots & \ddots & \ddots & & & & \\
\ddots & 0 & 15 & \ddots & & & \\
\ddots & 0 & 0 & 0 & \ddots & & \\
\ddots & 5 & 130 & 0 & 15 & \ddots & \\
\ddots & 0 & 0 & 0 & 0 & \ddots & \\
\ddots & 51 & -207 & 5 & 130 & 0 & \ddots \\
\ddots & -16 & 0 & 0 & 0 & 0 & \ddots \\
\ddots & 51 & -900 & 51 & -207 & 5 & \ddots \\
\ddots & 0 & 0 & -16 & 0 & 0 & \ddots \\
\ddots & 5 & -207 & 51 & -900 & 51 & \ddots \\
\ddots & 0 & 0 & 0 & 0 & -16 & \ddots \\
& \ddots & 130 & 5 & -207 & 51 & \ddots \\
& \ddots & 0 & 0 & 0 & 0 & \ddots \\
& \ddots & 15 & 0 & 130 & 5 & \ddots \\
& & \ddots & \ddots & \ddots & \ddots & \ddots
\end{array}\right],
$$

while the product of matrices $\left[P^{L} \mid Q^{L}\right]^{\prime-1} \cdot G^{L}$ turns out to have the following form:

$$
\left[\begin{array}{ccccccc}
\ddots & \ddots & \ddots & & & & \\
\ddots & 0 & 9 & \ddots & \ddots & & \\
\ddots & 3 & 0 & 0 & 9 & \ddots & \\
\ddots & 3 & -82 & 3 & 0 & 0 & \ddots \\
\ddots & 0 & 0 & 3 & -82 & 3 & \ddots \\
\ddots & 0 & 9 & 0 & 0 & 3 & \ddots \\
\ddots & 0 & 0 & 0 & 9 & 0 & \ddots \\
\ddots & 0 & 0 & 0 & 0 & 0 & \ddots \\
\ddots & 0 & 0 & 0 & 0 & 0 & \ddots \\
\ddots & 0 & 6 & 0 & 0 & 0 & \ddots \\
\ddots & 2 & -14 & 0 & 6 & 0 & \ddots \\
\ddots & 0 & -14 & 2 & -14 & 0 & \ddots \\
\ddots & 0 & 6 & 0 & -14 & 2 & \ddots \\
& \ddots & 0 & 0 & 6 & 0 & \ddots \\
& & \ddots & \ddots & \ddots & \ddots & \ddots
\end{array}\right]
$$

From equality (9) it follows that to find the matrix $R^{L}=\left[P^{L} \mid Q^{L}\right]^{-1} \cdot G^{L}$, it is required to apply to the rows of the matrix (12) an inverse permutation, that is, a permutation in which the records of the image and the inverse image are interchanged. From this, we obtain the required in (7) representation of the matrix $R^{L}$.

\section{The wavelet interpolation algorithm}

Let on the finite segment $\left[0,2^{L}\right]$ the values of the observation results are equal to zero at the ends. Then the left basic functions have the forms [25]

$$
\begin{aligned}
\varphi_{b 1}(t)= & \frac{7}{4} t_{+}^{3}-\frac{9}{2} t_{+}^{2}+3 t_{+}^{1}-2(t-1)_{+}^{3}, \\
& 0 \leq t \leq 2, \\
\varphi_{b 2}(t)= & \frac{3}{2} t_{+}^{2}-\frac{11}{12} t_{+}^{3}+\frac{3}{2}(t-1)_{+}^{3}-\frac{3}{4}(t-2)_{+}^{3}, \\
& 0 \leq t \leq 3,
\end{aligned}
$$

and they satisfy the calibration relations

$$
\begin{array}{cc}
\varphi_{b 1}(t)= & \frac{1}{2} \varphi_{b 1}(2 t)+\frac{3}{4} \varphi_{b 2}(2 t)+\frac{3}{16} \varphi_{3}(2 t), \\
\varphi_{b 2}(t)= & \frac{1}{4} \varphi_{b 2}(2 t)+\frac{11}{16} \varphi_{3}(2 t)+ \\
& +\frac{1}{2} \varphi_{3}(2 t-1)+\frac{1}{8} \varphi_{3}(2 t-2) .
\end{array}
$$

As to boundary basic wavelets, we use the cubic wavelets that are orthogonal to all third-degree polynomials [25], 


$$
\begin{array}{r}
w_{b 1}(t)=6 \varphi_{b 1}(2 t)-\frac{57}{5} \varphi_{b 2}(2 t)+\frac{919}{100} \varphi_{3}(2 t)- \\
-\frac{116}{25} \varphi_{3}(2 t-1)+\varphi_{3}(2 t-2), \\
w_{b 2}(t)=\frac{7}{3} \varphi_{b 2}(2 t)-\frac{319}{60} \varphi_{3}(2 t)+\varphi_{3}(2 t-3)+ \\
+\frac{101}{15} \varphi_{3}(2 t-1)-\frac{25}{6} \varphi_{3}(2 t-2) .
\end{array}
$$

They have the following supports

$$
\operatorname{supp} w_{b 1}=[0,3], \operatorname{supp} w_{b 2}=[0,3.5],
$$

and, accordingly, they have four zero moments

$$
\int_{0}^{3} x^{k} w_{b 1}(x) d x=\int_{0}^{3.5} x^{k} w_{b 2}(x) d x=0,
$$

for $k=0,1,2,3$.

At the right end of the segment, the basic functions mirror the functions $\varphi_{b 1,2}(t), w_{b 1,2}(t)$. As a result, for any grid $\Delta^{L}, L \geq 2$, a third-degree spline with zero boundary conditions can be represented as

$$
\begin{array}{r}
s^{L}(v)=c_{-2}^{L} \varphi_{b 1}(v)+c_{-1}^{L} \varphi_{b 2}(v)+ \\
+\sum_{i=0}^{2^{L}-4} c_{i}^{L} \varphi_{3}(v-i)+c_{2^{L}-3}^{L} \varphi_{b 2}\left(2^{L}-v\right)+ \\
+c_{2^{L}-2}^{L} \varphi_{b 1}\left(2^{L}-v\right), 0 \leq v \leq 2^{L},
\end{array}
$$

where the coefficients $c_{i}^{L} \forall i$ are the solution, for example, of the interpolation problem:

$$
\begin{aligned}
s^{L}(i) & =f(i), i=1,2, \ldots, 2^{L}-1, \\
\left(s^{L}\right)^{\prime}(i) & =f^{\prime}(i), i=1,2^{L}-1 .
\end{aligned}
$$

To prepare the system (5) for odd-even splitting we need to solve the system (11) for indices $j=$ $-2,-1, \ldots, 4$ with the following matrix

$$
\left[P^{L} \mid Q^{L}\right]_{-2,-1, \ldots, 4}^{\prime}=\frac{1}{8} \text {. }
$$

$$
\cdot\left[\begin{array}{ccccccccc}
4 & 48 & 0 & 0 & & & & & \\
6 & -\frac{456}{5} & 2 & \frac{56}{3} & 0 & & & & \\
\frac{3}{2} & \frac{1838}{25} & \frac{11}{2} & -\frac{638}{15} & 1 & 1 & & & \\
0 & -\frac{928}{2} 5 & 4 & \frac{808}{15} & 4 & -6 & & & \\
0 & 8 & 1 & -\frac{100}{3} & 6 & 15 & 1 & 1 & \\
0 & 0 & 0 & 8 & 4 & -20 & 4 & -6 & \\
& 0 & 1 & 15 & 6 & 15 & 1 & 1 & \\
& 0 & 0 & -6 & 4 & -20 & 4 & -6 & \\
& & & 1 & 1 & 15 & 6 & 15 & 1 \\
& & & 0 & 0 & -6 & 4 & -20 & 4 \\
& & & & & 1 & 1 & 15 & 6 \\
& & & & & 0 & 0 & -6 & 4 \\
& & & & & & & 1 & 1
\end{array}\right]
$$

provided that the equations corresponding to the zero rows of the matrix $G^{L}$ are removed from the system. This system is solvable and underdetermined. Therefore, we can choose any non-trivial solutions that interest us, for example:

1) $r_{-2}=r_{-1}=r_{0}=r_{1}=r_{6}=r_{9}=0$;

$r_{2}=240 ; r_{3}=-160 ; r_{4}=728 ;$

$r_{5}=-112 ; r_{7}=48 ; r_{8}=-72 ;$

$$
g_{-2}=g_{-1}=g_{1}=g_{3}=
$$$$
g_{5}=g_{7}=g_{9}=g_{11}=0 \text {; }
$$

$g_{0}=50 ; g_{2}=585 ; g_{4}=1080$;

$g_{6}=222 ; g_{8}=-130 ; g_{10}=-15 ;$

2) $\quad r_{-2}=90600 ; r_{-1}=-7550$;

$r_{0}=-376960 ; r_{1}=-25620$;

$$
r_{2}=1102416
$$

$r_{3}=-300336 ; r_{4}=450504$;

$$
r_{5}=r_{6}=r_{7}=r_{8}=0
$$

$g_{-2}=g_{-1}=g_{0}=g_{1}=g_{3}=g_{5}=$

$=g_{7}=g_{8}=g_{9}=g_{10}=g_{11}=0$;

$g_{2}=1498335$;

3)

$$
g_{4}=1038810 ; g_{6}=93855 \text {; }
$$

$r_{-2}=16360 ; r_{-1}=1290$;

$r_{1}=1044 ; r_{2}=-2088$;

$$
r_{0}=r_{3}=r_{4}=r_{5}=
$$$$
=r_{6}=r_{7}=r_{8}=0 \text {; }
$$

$g_{-2}=15920 ; g_{0}=9111$;

$g_{2}=-4626 ; g_{4}=-261 ;$

$$
g_{-1}=g_{1}=g_{3}=g_{5}=g_{6}=g_{7}=
$$$$
=g_{8}=g_{9}=g_{10}=g_{11}=0 \text {; }
$$

4)

$$
\begin{gathered}
r_{-2}=908 ; r_{-1}=75 ; r_{0}=696 ; \\
r_{1}=r_{2}=r_{3}=r_{4}=r_{5}= \\
\quad=r_{6}=r_{7}=r_{8}=0 ; \\
g_{-2}=904 ; g_{0}=1338 ; g_{2}=162 ; \\
g_{-1}=g_{1}=g_{3}=g_{4}=g_{5}=g_{6}= \\
=g_{7}=g_{8}=g_{9}=g_{10}=g_{11}=0 .
\end{gathered}
$$

So, the first seven colomns of the matrix $G^{L}$ are 


$\left[\begin{array}{ccccccc}15920 & -8 & 904 & 0 & 0 & 0 & 0 \\ 0 & -12 & 0 & 0 & 0 & 0 & 0 \\ 9111 & -3 & 1338 & -15 & 0 & 5 & 50 \\ -4626 & 0 & 162 & -3 & 1498335 & 51 & 585 \\ 0 & 0 & 0 & 0 & 0 & -16 & 0 \\ -261 & 0 & 0 & 0 & 1038810 & 51 & 1080 \\ 0 & 0 & 0 & 0 & 0 & 0 & 0 \\ 0 & 0 & 0 & 0 & 9385 & 5 & 222 \\ 0 & 0 & 0 & 0 & 0 & 0 & 0 \\ 0 & 0 & 0 & 0 & 0 & 0 & -130 \\ 0 & 0 & 0 & 0 & 0 & 0 & -15 \\ 0 & 0 & 0 & 0 & 0 & 0 & 0 \\ \vdots & \vdots & \vdots & \vdots & \vdots & \vdots & \vdots\end{array}\right]$

From the structure of the matrix $G^{L}$ it immediately follows, that the values of $\mathbf{h}^{L}$ at odd nodes are calculated from the explicit equations

$$
\frac{c_{i}^{L}}{h_{i}}=\left\{\begin{array}{l}
-12, \quad i=-1,1,2^{L}-5,2^{L}-3 ; \\
-16, \quad i=3,5, \ldots, 2^{L}-7,
\end{array}\right.
$$

while for the values of $\mathbf{h}^{L}$ at even nodes a system of linear equations is solved:

$$
\begin{aligned}
& 15920 h_{-2}+904 h_{0}=r_{-2}, \\
& 9111 h_{-2}+1338 h_{0}+50 h_{4}-15 h_{6}=r_{0}, \\
& -4626 h_{-2}+162 h_{0}+1498335 h_{2}+585 h_{4}-130 h_{6}-15 h_{8}=r_{2} \text {, } \\
& -261 h_{-2}+1038810 h_{2}+1080 h_{4}+207 h_{6}-130 h_{8}-15 h_{10}=r_{4} \text {, } \\
& 93855 h_{2}+222 h_{4}+900 h_{6}+207 h_{8}-130 h_{10}-15 h_{12}=r_{6}, \\
& -130 h_{4}+207 h_{6}+900 h_{8}+207 h_{10}-130 h_{12}-15 h_{14}=r_{8}, \\
& i=10,12, \ldots, 2^{L}-14: \\
& \begin{array}{r}
-15 h_{i-6}-130 h_{i-4}+207 h_{i-2}+900 h_{i}+ \\
+207 h_{i+2}-130 h_{i+4}-15 h_{i+6}
\end{array} \\
& i=2^{L}-12 \text { : } \\
& -15 h_{i-6}-130 h_{i-4}+207 h_{i-2}+900 h_{i}+207 h_{i+2}-130 h_{i+4} \\
& i=2^{L}-10 \text { : } \\
& -15 h_{i-6}-130 h_{i-4}+207 h_{i-2}+900 h_{i}+222 h_{i+2}+93855 h_{i+4} \\
& \left.i=2^{L}-8:\right\}=r_{i} \text {. } \\
& \begin{aligned}
-15 h_{i-6}-130 h_{i-4}+207 h_{i-2}+1080 h_{i}+1038810 h_{i+2} & -261 h_{i+6} \\
i & =2^{L}-6:
\end{aligned} \\
& -15 h_{i-6}-130 h_{i-4}+585 h_{i-2}+1498335 h_{i}+162 h_{i+2}-4626 h_{i+4} \\
& i=2^{L}-4 \text { : } \\
& -15 h_{i-6}+50 h_{i-4}+1338 h_{i}+9111 h_{i+2} \\
& i=2^{L}-2 \text { : } \\
& 904 h_{i-2}+15920 h_{i}
\end{aligned}
$$

Here the right-hand sides of the equations (13) are calculated from the formulas

$$
\begin{aligned}
r_{-2}= & c_{-2}^{L}+8 h_{-1}-4 h_{1} \\
r_{0}= & c_{0}^{L}+3 h_{-1}+15 h_{1}-5 h_{3}, \\
r_{2}= & c_{2}^{L}+3 h_{1}-51 h_{3}-5 h_{5} \\
r_{4}= & c_{4}^{L}-51 h_{3}-51 h_{5}-5 h_{7} \\
r_{i}= & c_{i}^{L}-5 h_{i-3}-51 h_{i-1}-51 h_{i+1}-5 h_{i+3}, \\
& i=6,8, \ldots, 2^{L}-10
\end{aligned}
$$

$$
r_{i}=\left\{\begin{array}{l}
c_{i}^{L}-5 h_{i-3}-51 h_{i-1}-51 h_{i+1}, \\
i=2^{L}-8, \\
c_{i}^{L}-5 h_{i-3}-51 h_{i-1}+3 h_{i+1}, \\
i=2^{L}-6, \\
c_{i}^{L}-5 h_{i-3}+15 h_{i-1}+3 h_{i+1}, \\
i=2^{L}-4 \\
c_{i}^{L}-4 h_{i-3}+8 h_{i-1}, \\
i=2^{L}-2 .
\end{array}\right.
$$

Note that the matrix of the system (13) has a strict diagonal dominance [26, p. 78] over the columns of the system. Such matrices are not degenerate, and therefore the system of equations has a unique solution, which is found by the sweep method with a guar- 
antee of the stability of the calculations.

Multiplying the matrices $\left[P^{L} \mid Q^{L}\right]^{-1}$ and $G^{L}$ we can recheck the analytical evaluation provided to ob- tain that the matrix $R^{L}$ is composed of two blocks according to $2^{L-1}+1$ basic spline functions of $V_{L-1}$ and $2^{L-1}$ basic wavelets of $W_{L-1}$ :

$$
\left[\begin{array}{ccccccc|cc|c}
16360 & -16 & 908 & 8 & 90600 & 0 & 0 & 0 & 0 & \\
0 & 0 & 696 & -24 & -376960 & 0 & 0 & 0 & 0 & \\
-2088 & 0 & 0 & 0 & 1102416 & 24 & 240 & 0 & -72 & \ddots \\
0 & 0 & 0 & 0 & 450504 & 24 & 728 & 24 & 0 & \ddots \\
0 & 0 & 0 & 0 & 0 & 0 & 0 & 24 & 656 & \ddots \\
0 & 0 & 0 & 0 & 0 & 0 & -72 & 0 & 0 & \ddots \\
0 & 0 & 0 & 0 & 0 & 0 & 0 & 0 & -72 & \ddots \\
\vdots & \vdots & \vdots & \vdots & \vdots & \vdots & 0 & 0 & 0 & \ddots \\
& & & & & & \vdots & \vdots & \ddots & \ddots \\
1290 & 0 & 75 & 0 & -7550 & 0 & 0 & 0 & 0 & \\
1044 & 0 & 0 & 0 & -25620 & 0 & 0 & 0 & 0 & \\
0 & 0 & 0 & 0 & -300336 & -16 & -160 & 0 & 48 & \ddots \\
0 & 0 & 0 & 0 & 0 & 0 & -112 & -16 & -112 & \ddots \\
0 & 0 & 0 & 0 & 0 & 0 & 48 & 0 & -112 & \ddots \\
0 & 0 & 0 & 0 & 0 & 0 & 0 & 0 & 48 & \ddots \\
\vdots & \vdots & \vdots & \vdots & \vdots & \vdots & 0 & 0 & 0 & \ddots \\
& & & & & & \vdots & \vdots & \ddots & \ddots
\end{array}\right] .
$$

Here diagonal points mean that the preceding two columns are repeated the appropriate number of times while going each time two positions right and moving one position down. The last seven columns of both blocks of the matrix $R^{L}$ mirror the first seven columns; the empty positions of matrices are equal to zero.

As a result, the values of the spline coefficients on the thinned grid and the wavelet coefficients are calculated by the formulas (7). Thus, the operation of wavelet decomposition can be performed without explicit representation and the use of a filter block.

The mathematical complexity of the algorithm for solving system (13) by the sweeping method is valued by the number of required arithmetic operations [26, p. 100]: $15 \cdot\left(2^{L-1}-1\right)$ additions, $15 \cdot\left(2^{L-1}-1\right)$ multiplications, and $4 \cdot\left(2^{L-1}-1\right)+1$ divisions. Calculating the right-hand sides of the equations requires $5 \cdot\left(2^{L-1}-2\right)$ multiplications and $4 \cdot\left(2^{L-1}-2\right)$ additions; obtaining the spline-coefficients at the nodes of the sparse grid requires $2 \cdot\left(2^{L-1}-1\right)$ additions and $3 \cdot\left(2^{L-1}-4\right)+10$ multiplications. The calculation of the wavelet-coefficients: $3 \cdot\left(2^{L-1}-4\right)+14$ multipli- cations and $2 \cdot\left(2^{L-1}-4\right)+10$ additions. If we make no differences between the arithmetic operations, then the total number of such operations for one step of the wavelet decomposition is approximately $53 \cdot 2^{L-1}$.

\section{The algorithm of wavelet analysis}

consists of performing the following steps:

\subsection{On the finite segment $[a, b]$}

the values of the observation results are reset to zero at the ends by subtracting values of the line

$$
f(a)+(x-a)(f(b)-f(a)) /(b-a)
$$

from the entire time series [24].

\subsection{The wavelet interpolation algorithm}

for a given $L$ is incorporated.

7.3 If $L \geq 2$,

then the value of $L$ decreases by 1 , and the algorithm goes to step 2. 


\subsection{Otherwise, at each level $L$}

of the decomposition, the rejection of insignificant wavelet coefficients is performed according to some criterion [3, 17], and the spline coefficients are sequentially restored according to the moving average algorithm (4).

\subsection{After wavelet analysis}

of the differences obtained at the first stage of the algorithm and reconstructing (of course, with some approximation) the spline coefficients for the densest mesh, the values of the line (14) are added to values of the approximating spline of the third degree.

\section{An example}

The results of numerical experiments using the proposed algorithm were presented earlier in [24]. Let $L=4$ and the discrete signal be represented as values of the analytic function $f(x)=\left(x^{2}-16\right)^{2}$, given at the points $\Delta^{4}: x=-4,-3.5, \ldots, 4$. Since at the extreme points of $x= \pm 4$, the homogeneous boundary conditions necessary for constructing the wavelet decomposition are satisfied, there is everything to investigate the application of the above wavelets to the problem of the wavelet analysis. After solving the corresponding interpolation problems and sequentially executing the wavelet analysis procedure, at the last stage $L=2$, we reject all the wavelet expansion coefficients obtained.

In this case, let the mean square error of approximation be estimated by the expression

$$
\operatorname{MSE}=\left(\frac{1}{15} \sum_{i=1}^{15}\left(f\left(x_{i}\right)-S^{2}\left(x_{i} / 2+2\right)\right)^{2}\right)^{1 / 2} .
$$

Table 1 presents four calculation variants: 1) from the solution of the interpolation problem, I; 2) according to the scheme presented in this article, $6 ; 3$ ) using wavelets orthogonal to the third degree polynomials $[19,25], 4 ; 4)$ using interpolation cubic wavelets [16], ICW.

Table 1: Comparative characteristics of the wavelet processing algorithms

\begin{tabular}{|ccccc|}
\hline & I & 6 & 4 & ICW \\
\hline$\left(S^{2}\right)^{\prime \prime}(-4)$ & 127.5 & 117.75 & 118.1172 & 120 \\
\hline$\left(S^{2}\right)^{\prime \prime}(0)$ & -64.4998 & -72.29 & -72.0548 & -71.9999 \\
\hline MSE & 0 & 0.323 & 0.3321 & 0.66 \\
\hline
\end{tabular}

Thus, in this example, the use of a more complicated approximation scheme with six zero moments leads to an increase in the approximation accuracy, but an increase of a distance from the true values of the approximated derivative. The algorithm built based on the interpolation of cubic spline wavelets with the property of the best mean-square approximation of the second derivative of the function being approximated, is in general, inferior in all indicators to algorithms that have zero moments.

\section{Conclusion}

The article discusses the further development of the author's procedure [18] for an even-odd partition of the defining system of the Hermite wavelet expansion for the practically important case of approximating functions that do not require specifying the values of the derivatives, based on $B$-splines of the third degree.

The advantage of the new algorithm is its stability and simplicity of realization because of a matrix possessing strict diagonal prevalence is solved at each step of convolution.

The directions of our future research consist in the extension of the proposed approach to splines of a higher degree and a larger number of zero moments which can provide new opportunities for the development of algorithms for constructing and applying spline wavelets.

\section{References:}

[1] I. Daubechies, Ten lectures on Wavelets, Society for Industrial and Applied Mathematics, Philadelphia (PA), 1992.

[2] S. Mallat, A Wavelet Tour of Signal Processing, Academic Press, San Diego (CA), 1999.

[3] E.J. Stollnitz, T.D. DeRose, D.H. Salesin, Wavelets for Computer Graphics, Morgan Kaufmann Publishers, San Francisco, 1996.

[4] C.K. Chui, An Introduction to Wavelets, Academic Press, New York, London, 1992.

[5] V. Apetrei, C. Filote, A. Graur, The analysis of the deforming regime generated by AC-DC converters using discrete wavelet transform, WSEAS Transactions on Systems, Vol.13, No.1, 2014, pp. 551-559.

[6] M.W. Frazier, An Introduction to Wavelets through Linear Algebra, Springer-Verlag, New York, 1999.

[7] M.F. Pouyani, M. Vali, M.A. Ghasemi, Lung sound signal denoising using discrete wavelet transform and artificial neural network, Biomedical Signal Processing and Control, Vol.72, 2022, article No. 103329 
[8] M. Luo, L. Ge, Z. Xue, J. Zhang, Y. Li, X. Xiao, Research on De-noising of downhole engineering parameters by wavelet based on improved threshold function, International Journal of Circuits, Systems and Signal Processing, Vol.15, 2021, pp. 722-729.

[9] X. Li, S. Zhang, H. Zhao, A fast image compression algorithm based on wavelet transform, International Journal of Circuits, Systems and Signal Processing, Vol.15, 2021, pp. 809-819.

[10] Q. Zhang, Y. Li, Medical image segmentation algorithm based on multi-scale color wavelet texture, International Journal of Circuits, Systems and Signal Processing, Vol.15, 2021, pp. 928935.

[11] P. Vonghirandecha, P. Bhurayanontachai, S. Kansomkeat, S. Intajag, No-reference retinal image sharpness metric using daubechies wavelet transformInternational, Journal of Circuits, Systems and Signal Processing, Vol.15, 2021, pp. 1064-1071.

[12] T. Tuncer, S. Dogan, P. Plawiak, A. Subasi, A novel Discrete Wavelet-Concatenated Mesh Tree and ternary chess pattern based ECG signal recognition method, Biomedical Signal Processing and Control, Vol.72, 2022, article No. 103331.

[13] A. Cohen, I. Daubechies, J.C. Feauveau, Biorthogonal bases of compactly supported wavelets, Communications on Pure and Applied Mathematics, Vol.45, 1992, pp. 485-560.

[14] A. Cohen, I. Doubeshies, P. Vial, Wavelets on the interval and fast wavelet transforms, Applied and Computational Harmonic Analysis, Vol.1, 1993, pp. 54-81.

[15] M. Lyche, K. Mǿrken, E. Quak, Theory and algorithms for non-uniform spline wavelets, Multivariate Approximation and Applications, eds. N. Dyn, D. Leviatan, D. Levin, A. Pinkus, Cambridge University Press, Cambridge, 2001, pp. 152-187.

[16] J. Wang, Cubic spline wavelet bases of sobolev spaces and multilevel interpolation, Applied and Computational Harmonic Analysis, Vol.3, No.2, 1996, pp. 154-163.

[17] R. Wilson, Multiresolution image modeling, Electronics and Communications Engineering Journal, Vol.9, No.2, 1997, pp. 90-96.
[18] B.M. Shumilov, A splitting algorithm for wavelet transforms of the Hermite splines of the seventh degree, Numerical Analysis and Applications, Vol.8, No.4, 2015, pp. 365-377.

[19] B.M. Shumilov, On splitting for cubic spline wavelets with four zero moments on an interval, Journal of Computational Technologies, Vol.26, No.2, 2021, pp. 72-87.

[20] C. De Boor, A Practical Guide to Splines, Applied Mathematical Sciences, Vol.27, SpringerVerlag, New York, 1978.

[21] K. Koro, K. Abe, Non-orthogonal spline wavelets for boundary element analysis, Engineering Analysis with Boundary Elements, Vol.25, 2001, pp. 149-164.

[22] B.M. Shumilov, Splitting algorithms for the wavelet transform of first-degree splines on nonuniform grids, Computational Mathematics and Mathematical Physics, Vol.56, No.7, 2016, pp. 1209-1219.

[23] S. Pissanetzky, Sparse Matrix Technology, Academic Press, London, 1984.

[24] B.M. Shumilov, On five-diagonal splitting for cubic spline wavelets with six vanishing moments on a segment, WSEAS Transactions on Information Science and Applications, Vol.17, 2021, pp. 156-165.

[25] D. Cerná, Cubic spline wavelets with four vanishing moments on the interval and their applications to option pricing under Kou mode, International Journal of Wavelets, Multiresolution and Information Processing, Vol.17, No.1, 2019, article No. 1850061.

[26] A.A. Samarskii, E.S. Nikolaev, Numerical Methods for Grid Equations, Vol. I Direct Methods, Birkhauser, Basel, 1989.

\section{Creative Commons Attribution License 4.0 (Attribution 4.0 International , CC BY 4.0)}

This article is published under the terms of the Creative Commons Attribution License 4.0

https://creativecommons.org/licenses/by/4.0/deed.en US 